\title{
Z Resesach Square \\ Identification of TUBB4A as a Prognostic Biomarker of Melanoma by Transcriptomic Data and in vitro Experiments
}

\section{Jiaqi Zhang}

The Affiliated Changzhou No 2 People's Hospital of Nanjing Medical University https://orcid.org/00000002-3580-9227

\section{Zhe Li}

Shuguang Hospital Affiliated to Shanghai University of Traditional Chinese Medicine

Hui Liu

Nanjing Tech University

Qi Wu

Heze Medical College

\section{Zhiqiang Sun}

The Affiliated Changzhou No 2 People's Hospital of Nanjing Medical University

\section{Yan Cao}

The Affiliated Changzhou No 2 People's Hospital of Nanjing Medical University

\section{Wei Gao}

The Affiliated Changzhou No 2 People's Hospital of Nanjing Medical University Judong Luo ( $\square$ judongluo@suda.edu.cn )

The Affiliated Changzhou No 2 People's Hospital of Nanjing Medical University https://orcid.org/00000002-7355-7374

\section{Xuejun Liu}

Nanjing Tech University

\section{Primary research}

Keywords: Melanoma, TUBB4A, Prognostic biomarker, Tubulin, Differential expression

Posted Date: November 2nd, 2021

DOI: https://doi.org/10.21203/rs.3.rs-1013292/v1

License: (c) (1) This work is licensed under a Creative Commons Attribution 4.0 International License. Read Full License 


\section{Abstract \\ Background}

Melanoma is one of the most malignant skin carcinomas with high metastatic potential. Increasing evidences have demonstrated that $\beta$-tubulin 4A (TUBB4A) play key role in development and progression of several types of human cancer. However, the potential function of TUBB4A in cutaneous melanoma remains to be determined.

\section{Methods}

We first performed differential expression analysis between skin melanoma tissues and normal tissues from GEO and TCGA datasets, and then ran survival analysis to identify prognostic-related key genes. We went further to conduct the verification of in vitro biochemical experiments to explore the functional roles of key gene TUBB4A. Two small molecule inhibitors of TUBB4A, Dihydroartemisinin (DHA) and Nocodazole, were used to examined the effect on apoptosis and cell cycle progression of melanoma cells.

\section{Results}

We found that TUBB4A is markedly correlated to the overall survival of cutaneous melanoma patients. The co-expressed genes with TUBB4A are enriched in the melanoma-related pathways and function. Then, the experimental results showed that knockdown of TUBB4A inhibit the proliferation and migration of A375 and B16-F10 melanoma cells. Moreover, two small molecular agents targeting TUBB4A, Dihydroartemisinin and Nonocodazole, dpromote the apoptosis of melanoma cells and made the tumor cells significantly blocked in G2/M stage.

\section{Conclusion}

We identified and validated in vitro that TUBB4A may be a prognostic biomarker and therapeutic target for melanoma.

\section{Background}

Malignant melanoma is the most lethal form of skin cancer and its incidence rates have been rising rapidly over the past few decades. Approximate 232,100 cases $(1.7 \%)$ of all newly diagnosed primary malignant tumors (excluding non-melanoma skin cancer) are skin cutaneous melanoma (SKCM) for each year. There are about 55,500 cancer deaths ( $0.7 \%$ of all cancer deaths) due to skin melanoma [1], and $10 \%$ melanoma cases are diagnosed at advanced stage and suffer from poor prognosis [2]. As its strong invasion and metastasis, identification of novel biomarker is essential to the early diagnosis and 
treatment of melanoma. Some of the clinical biomarkers have been reported, including S100, HMB 45, Melan A, CSPG4 and so on [3-6]. For example, Bastian et al. reported that the occurrence of oncogenic mutants leads to malfunction involved in driver genes, such as NRAS, HRAS, BRAF, KIT and GNA11 [7, 8]. Also, loss of function of tumor suppressor genes, such as CDKN2A, TP53 and PTEN, can also be used for clinical diagnosis and prognosis of melanoma [8]. Although the treatment of metastatic melanoma has been greatly improved by targeted and immune therapies, identification of novel biomarkers of SKCM metastasis is crucial for development of diagnostic and therapeutic strategies.

Microtubules are rigid and hollow fibers composed of the cell cytoskeleton, and play an important role in the maintenance of cell shape, cell motility and cell cycle [9]. Altered microtubules dynamics is a hallmark of carcinogenesis, and overexpression of $\beta$-tubulins is associated to the progression, prognosis and resistance to chemotherapeutic agents in different cancers [10]. For example, it is reported that $\beta$ III and $\beta I V$-tubulins are highly expressed in pancreatic tumors, compared to normal pancreas tissue [11]. Haider et al. also reported that the aberrant expression of the $\beta$-tubulins is commonly observed in metastatic cancer cells [12].

TUBB4A is a member of the $\beta$-tubulin family and encodes $\beta$-tubulin 4A. $\beta$-tubulin is involved in several intracellular processes like mitosis, motility and transport [13]. Previous studies have shown that GLUT1 and its binding partner TUBB4 as potentially druggable targets in glioblastoma multiforme. Silencing TUBB4 reduces glioblastoma stem cell tumorsphere formation, self-renewal and proliferation in vitro [14]. The down-regulation of $\beta I V a-t u b u l i n$ in lung cancer cells increases their sensitivity to tubulin-binding agents, such as vinorelbine, vincristine and paclitaxel [15]. Ross et al. explored the impact of hypoxia on the proteome, and found that TUBB4A differential expression primarily involved in structural and binding processes of the prostate cancer [16]. Atjanasuppat et al. also reported that ERK signaling mediates upregulation of $\beta I V a-t u b u l i n$ gene and confers paclitaxel resistance in $\mathrm{H} 460$ floating lung cancer cells [17]. However, the potential function of TUBB4A has not been reported in melanoma to date.

In this study, we conducted differential expression analysis on two large SKCM cohorts, and found that TUBB4A is significantly up-regulated in SKCM tissues compared to normal tissues. Significant correlation between TUBB4A expression and patient overall survival was observed. Moreover, our in vitro experiments showed that inhibition of TUBB4A reduce the migration and proliferation of melanoma cells. Two drugs reported to target TUBB4A in DrugBank, Dihydroartemisinin and Nocodazole, were evaluated by flow cytometry whether they promote the apoptosis and block cell cycle of SKCM cells. The assay results showed that both drugs significantly induced cell apoptosis and G2/M cell cycle arrest in the group received treatment of Dihydroartemisinin and Nocodazole. In summary, our study indicated that TUBB4A may serve as a promising novel biomarker for treating melanoma. Targeting TUBB4A will contribute to develop new therapeutic agents against melanoma.

\section{Materials And Methods}

\section{Gene expressions and differential expression analysis}


The gene expression profiles of SKCMs were downloaded from GEO (https://www.ncbi.nlm.nih.gov/geo/) and TCGA (http://cancergenome.nih.gov/) database [18]. For GEO, we selected

human melanoma datasets according to the criteria: sample size is more than 10 , both tumor and nontumor samples are included. As a result, three GEO datasets GSE46517, GSE15605 and GSE3189 are selected, and the combined dataset include 207 tumor samples and 31 normal samples. Details of the three datasets were listed in Table 1. The differential expression analysis by integration of TCGA and GTEx datasets, and identification of the differentially expressed genes (DEGs) between melanoma and normal samples was conducted as described in our previous study [19].

The Limma R package was used to conduct differential expression analysis[20]. The genes with $P$-value $<$ 0.05 and absolute fold change $>2$ were considered as differentially expressed genes (DEGs). The $R$ packages ggplot2 was used to draw the volcano plots of the DEGs. Venn diagram analyses were conducted to obtain the overlapping DEGs among the three datasets mentioned above.

\section{Enrichment, survival and co-expression analysis}

Enrichment analysis on Gene Ontology (GO) functional annotations and Kyoto Encyclopedia of Genes and Genomes (KEGG) pathways were performed on the set of overlapping DEGs using R package clusterProfiler [21]. All the three types of GO functional annotations, including biological process (BP), cellular composition (CC), and molecular function (MF), were covered.

Survival analysis was conducted on 458 SKCM patients with RNA-seq expression profiles and clinical information obtained from TCGA. The patients were divided into high and low groups by TUBB4A median expression levels. The prognostic effect of the expression of TUBB4A on overall survival (OS) and disease-free survival (DFS) were estimated using the Kaplan-Meier method and survival curves were evaluated using the log-rank test.

Co-expressed genes of TUBB4A were clustered and demonstrated by the heat map generated by LinkedOmics [22]. The top 50 positive and negative correlated genes with TUBB4A were identified by Pearson correlations ( $P$-value $<0.05)$.

\section{Cell lines and cell culture}

The commercially available melanoma cell lines A375 and B16-F10 were purchased from the Shanghai Cell Bank of the Chinese Academy of Sciences (Shanghai, China). A375 and B16-F10 cells were cultured in Dulbecco's modified Eagle's medium (DMEM) containing $10 \% \mathrm{FBS}$ at $37^{\circ} \mathrm{C}$ in a humidified incubator under $5 \% \mathrm{CO}_{2}$ condition.

\section{siRNA transfection}

The specific small interfering RNA was performed using custom-made siRNA targeting the TUBB4A mRNA region (siTUBB4A sense: GGAGGUUAUCAGUGACGAATT, siTUBB4A antisense: UUCGUCACUGAUAACCUCCTT) and negative control (siNC sense: UUCUUCGAACGUGUCACGUTT, 
antisense: ACGUGACACGUUCGGAGAATT) (GenePharma, China). Cells were cultured in a 6-well plate and transfected with lip3000 (Invitrogen, Carlsbad, CA, USA) following the instructions of the manufacturer. After $48 \mathrm{~h}$ of transfection, the cells were used for subsequent experiments.

\section{RNA isolation and quantitative real-time PCR (qRT-PCR)}

PrimeScript RT reagent kit with gDNA Eraser (Takara, Tokyo, Japan) is used to prepared for cDNA and real-time PCR was performed by using SYBR Green II Mixture (TaKaRa) according to the manufacturer's protocol. $18 \mathrm{~S}$ was used for normalization and the comparative $\mathrm{Ct}$ method $(\Delta \Delta \mathrm{Ct})$ was used to evaluate mRNA expression. The specific primer pairs were as follows: $18 \mathrm{~S}$ (internal control gene) primer (forward primer, 5'-GGAGAGGGAGCCTGAGAAACG-3'; reverse primer, 5'-TTACAGGGCCTCGAAAGAGTCC-3'); TUBB4A primer (forward primer, 5'-CCGGACAACTTCGTGTTTGG-3'; reverse primer, 5'TCGCGGATCTTACTGATGAGC-3').

\section{Cell proliferation assay}

For CCK8 proliferation assay, A375 and B16-F10 cells were transfected as previously described. Then, 800 cells were resuspended in $100 \mu \mathrm{L}$ DMEM supplemented with $10 \%$ FBS and then added to a 96 well plate. After 24h, 48h, 72h and 96h, cell proliferation was investigated using CCK8 (Dojindo Molecular Technologies, USA) according to the manufacturer's instruction.

\section{Wound healing and transwell assay}

Cells were seeded in a 6 -well plate and cultured for $48 \mathrm{~h}$ to reach approximately $100 \%$ confluence. The sterile pipette tip was used to scratch a linear wound and serum free DMEM was added for further culturing. Wound healing images were captured on $0 \mathrm{~h}, 24 \mathrm{~h}$ and $48 \mathrm{~h}$ using an inverted microscope. For transwell assay, $5 \times 10^{4}$ melanoma cells were seeded into the upper well (Corning, USA). The lower chamber was filled with $700 \mu \mathrm{L}$ of DMEM containing $20 \%$ FBS. Transwell chambers were placed in an incubator $\left(37^{\circ} \mathrm{C}, 5 \% \mathrm{CO}_{2}\right)$ for $12 \mathrm{~h}$. Cells in the upper chamber were fixed with $4 \%$ paraformaldehyde for 15 min, stained with $0.1 \%$ crystal violet for 15 min and counted under an inverted microscope.

\section{Drug treatments and flow cytometric analysis}

TUBB4A inhibitors were retrieved from DrugBank [23]. Two small molecule agents, Dihydroartemisinin (DHA) and Nocodazole, are reported to bind TUBB4A protein. Cells seeded in a 6-well plate were treated with Dihydroartemisinin $(20$ and $40 \mu \mathrm{M})$ for $48 \mathrm{~h}$ and Nocodazole $(0.075$ and $0.15 \mu \mathrm{M})$ for $24 \mathrm{~h}$

separately $[24,25]$. Annexin V-FITC/PI kit (Becton Dickinson, USA) were used to measure the apoptosis of melanoma cells. All operations were carried out strictly according to the manufacturer instruction. In brief, cells were harvested and washed with phosphate buffer saline (PBS), and resuspended in 1×binding buffer. Next, cells were stained with $5 \mu \mathrm{L}$ FITC-Annexin and $5 \mathrm{PI}$ for 15 min under dark conditions. Cell clumps were removed by passing-through a cell strainer. The apoptosis of cells was measured by FACS 
Calibur flow cytometer (BD Biosciences, USA). Early apoptosis and late apoptosis were summed and the total apoptosis rate was calculated.

A single-cell suspension was prepared, which were subsequently fixed with $70 \%$ ethanol at $4^{\circ} \mathrm{C}$ for $12 \mathrm{~h}$. The fixed cells were then stained with $500 \mu \mathrm{L}$ Working Solution $(0.02 \%$ RNase Solution, $0.02 \%$ PI Solution, $0.1 \%$ Assay Buffer) at $37^{\circ} \mathrm{C}$ for $30 \mathrm{~min}$ and $4^{\circ} \mathrm{C}$ for $30 \mathrm{~min}$. Cell clumps were removed by passing-through a cell strainer and data was acquired using BD Accuri Cytometry (BD BioSciences, USA).

\section{Statistical analysis}

Statistical analyses were performed using SPSS 23.0 software (IBM, Chicago, IL, USA) and GraphPad Prism 8.0 (GraphPad Software, La Jolla, CA, USA). Student's t test was used to analyze the differences between two groups. One-way analysis of variance (ANOVA) was used for the comparison among three or more groups. A $P$-value less than 0.05 was considered as statistical significance.

\section{Results}

\section{Dysregulated genes closely related to melanoma}

Differential expression analysis on were conducted on three GEO datasets independently, and the volcano plots of DEGs ( $P$-value $<0.05$, absolute fold change $>2)$ were displayed in Fig. 1A-C. Subsequently, DEGs common in three SKCM datasets are filtered out and illustrated in Venn diagram. As shown in Fig. 1D-F, there are 580 overlapping DEGs in all three datasets, in which 165 genes were upregulated and 393 genes were downregulated. Moreover, overexpression of TUBB4A was found in melanoma tissues compared with normal tissues in all three datasets (Fig. 1G-I).

To uncover the role of these differentially expressed genes in the pathogenesis of melanoma, GO and KEGG enrichment analysis was carried out. As shown in Fig. 2A-C, they are significantly enriched in skin epidermis-related biological processes, such as skin and epidermis development, epidermal cell and keratinocyte differentiation, and extracellular structure organization. As for cellular component, these DEGs are significantly enriched in the cell-cell junction, collagen-containing extracellular matrix, apical part of cell, cornified envelope, and intermediate filament. For molecular function, they are significantly enriched in cell adhesion molecular binding, actin binding, structure constituent of cytoskeleton, extracellular matrix structural constituent, and serine-type peptidase activity.

The KEGG enrichment analysis shows top 25 significantly enriched pathways are involved in the process of cancerogenesis (Fig. 2D). More specifically, up-regulated genes are mainly enriched in the pathways related to transcription misregulation in cancer, glycosphingolipid biosynthesis-lacto and neolacto series, p53 signaling pathway and melanogenesis. Down-regulated genes are significantly enriched in histidine metabolish, Staphylococcus aureus infection, amoebiasis, drug metabolism and metabolism of xenobiotics by cytochrome P450. 


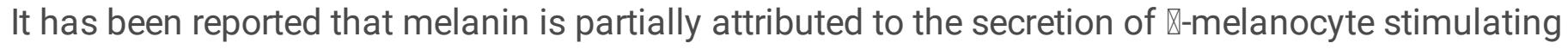
hormone ( $\mathbb{X}-\mathrm{MSH}$ ) induced by keratinocytes, while ultraviolet (UV) induction of $\mathbb{X}-\mathrm{MSH}$ in skin is directly regulated by p53 [26]. Key signal molecule p53 activated in response to DNA damage, trafficked in melanoma and thyroid cancer cells and was associated with cell proliferation and progression. Moreover, it also mediates cell-cycle arrest, apoptosis, and senescence [27].

\section{TUBB4A is over-expressed and correlated to prognosis in melanoma}

We conducted differential expression analysis by integration of TCGA and GTEx datasets. The differential expression analysis revealed 1,485 genes that are differentially expressed between tumor and normal samples. Among them, 514 genes are up-regulated and 972 genes are down-regulated. Since dysregulated genes are often main cancerogenic factors, we performed survival analysis on dysregulated genes independently. The Kaplan-Meier analysis results showed that five up-regulated genes are significantly related to the both overall survival and disease-free survival of cancer patients. They are TUBB4A, PSEN2, SLC45A2, QPRT and TRPV2. Table 2 listed the details of the five genes.

We have conducted extensive literature search to verify the genes associated to melanoma survival rate. SLC45A2 is involved in melanosome maturation and pigmentation and already reported to be associated with risk of cutaneous malignant melanoma $[28,29]$. The upregulation of PSEN2 is associated with human melanoma aggressiveness and poor prognosis, and it is also the target of MYC [30]. According to previous study [31], TRPV2 exhibited ectopic distribution both in melanocytes and melanoma cells. Moreover, activation of TRPV2 could lead to the decline of cell viability for melanoma A2058 and A375 cells [31].

Most importantly, TUBB4A is the gene with the most statistical significant in the prediction of patient survival status. The Kaplan-Meier survival curve showed that patients with high TUBB4A expression had significantly shorter overall survival and disease-free survival than those with low expression (Fig. 3A, B). Then, we preformed co-expression analysis of TUBB4A in SKCM cases through LinkedOmics in the TCGA database. As shown in Fig. 3C, D, fifty significantly co-expressed genes were demonstrated by heatmap, showing that they were positively or negatively correlated with TUBB4A.

\section{TUBB4A knockdown significantly inhibits SKCM cell proliferation}

To further explore the role of TUBB4A in SKCM, small interfering RNAs (siRNAs) to silence TUBB4A gene was transfected in A375 and B16-F10 cells. We ensure the transfection resulted in sufficient knockdown of TUBB4A expression. The downregulation at RNA level was evident at $48 \mathrm{~h}$ past transfection for both cell lines (Fig. 4A, B). Cell viability was determined using the CCK8 assay based on absorbance at $450 \mathrm{~nm}$ after 24h, 48h, 72h and 96h. Both A375 and B16-F10 cells with TUBB4A knockdown by siRNA showed reduced proliferation compared to the control group (si-NC, Fig. 4C, D). These results indicate that the higher expression of TUBB4A is closely associated to the proliferation of melanoma cells.

\section{TUBB4A knockdown reduce SKCM cell migration in vitro}


SKCM cell migration capacity was detected by wound healing assay. A375 and B16-F10 cells of which TUBB4A is knocked down by siRNA showed a larger open wound area compared with the control group after $24 \mathrm{~h}$ and $48 \mathrm{~h}$ (Fig. 5a, C). The difference in open wound area after $24 \mathrm{~h}$ and $48 \mathrm{~h}$ was quantified by calculating the percentage of change in the open wound area (Fig. 5B, D, $p<0.05$ ).

In addition, transwell assay is also used to evaluate its impact on migration capacity. Results demonstrated that TUBB4A knockdown caused a strong reduction in cell migration in A375 (t test, $P<0.05$ ) and B16-F10 (t test, $P<0.05$ ) cells, compared to the control group (Fig. 6A-D). These findings indicate that TUBB4A functions as a driver factor in increasing melanoma cell motility.

\section{TUBB4A inhibitors significantly induce apoptosis of melanoma cells}

We went further to search for small molecule drugs that target TUBB4A in the DrugBank database. There are five small molecule agents potentially target to TUBB4A. We selected two drugs, Dihydroartemisinin (DHA) and Nocodazole, which have been approved or already in experimental stage. Dihydroartemisinin is an artemisinin derivative and antimalarial agent used in the treatment of uncomplicated Plasmodium falciparum infections, and it has been also reported to bind to TUBB4A protein [32]. Nocodazole is a 16membered macrolide that mimics the biological effects of taxol and functions as inhibitor of microtubule function. Nocodazole has also been reported to inhibit the activity of TUBB4A [33].

Next, we went to test whether treatment with these two drugs separately can induce apoptosis of melanoma cell. As A375 is human melanoma cell line, we conducted assays on A375 cell line. The A375 cells were treated with DHA $(20 \mu \mathrm{M}$ and $40 \mu \mathrm{M})$ for $48 \mathrm{~h}$ and Nocodazole $(0.075 \mu \mathrm{M}$ and $0.15 \mu \mathrm{M})$ for $24 \mathrm{~h}$ separately. As shown in Fig. 7A, C, microscopy observations revealed that the exposure to DHA and Nocodazole triggered fragmentation in the cancer cells. The A375 cell apoptosis induced by these two agents was confirmed by staining with Annexin V-FITC/PI and subsequent analysis by flow cytometry. As shown in Fig. 7A-D, the proportion of apoptotic cells (quadrant Q2 An+/PI+-late apoptosis and quadrant Q4 An+/PI- -early apoptosis) increased significantly in a concentration-dependent manner in cell line after the exposure to two drugs. Our results indicate the pro-apoptotic activity of DHA and Nocodazole against A375 cells.

\section{TUBB4A inhibitors modulate melanoma cell cycle progression}

We also examined the effect of two TUBB4A inhibitors on cell cycle progression in A375 cells, using propidium iodide staining. The A375 cells were treated by DHA for $48 \mathrm{~h}$ and Nocodazole for $24 \mathrm{~h}$, respectively. With the increase of DHA concentration, the cell cycle of A375 cells (Fig. 8A, B) was significantly blocked in G2/M phase. As shown in Fig. 8C, D, Nocodazole exerted significant tumor cell cycle arrest, and the dosages effect of $0.075 \mu \mathrm{M}$ and $0.15 \mu \mathrm{M}$ differ slightly. The results revealed that DHA and Nocodazole inhibited the proliferation of A375 cells by inducing cell cycle arrest.

\section{Discussion}


Despite the remarkable advancement made in melanoma therapeutic strategies, the long-term prognosis of melanoma patients remains poor due to limited understanding of the underlying mechanisms of tumor initiation and development. This study aimed to explore the function and significance of TUBB4A and its inhibition agents in cutaneous melanoma. We demonstrated that TUBB4A was highly expressed in SKCM, and the results of survival analysis showed that the expression level of TUBB4A have significant effects on the viability of melanoma cells. Next, we validated and confirmed the functions of TUBB4A in tumorigenesis and development in melanoma. TUBB4A knockdown by siRNA in the A375 and B16-F10 melanoma cells significantly inhibited the proliferation and metastasis of cancer cell. Finally, we found that two TUBB4A inhibitors, Dihydroartemisinin and Nocodazole, promoted the cell apoptosis and induced cell cycle arrest in G2/M phase in vitro.

Class III beta-tubulin ( $\beta 3$-tubulin) has been reported that it correlates with enhanced neoplastic cell survival, metastasis and resistance to chemotherapy [34]. But there are few studies focusing on the role of Class IV beta-tubulin ( $\beta 4$-tubulin) in tumor progression. Previous studies have provided the first evidence that the upregulation of TUBB4 and TUBB3 is coupled with increased cell migration in endothelial-mesenchymal transition-induced human microvascular endothelial cells (HMEC-1) [35]. Also, TUBB4 are necessary for the transport and proper localization of $\mathrm{N}$-cadherin within the plasma membrane [35]. Moreover, non-adherent culture induces paclitaxel resistance in $\mathrm{H} 460$ lung cancer cells via ERK-mediated up-regulation of bIVa-tubulin [17]. Above studies have shown that TUBB4A plays crucial role in tumorigenesis. Thus, it is of significance to explore the role of TUBB4A in cancer metastasis, as well as its potential functions in the acquirement of drug resistance.

Furthermore, we explored the the transcription factors (TFs) of TUBB4A, and retrieved four TFs from KnockTF database [36], including HOXA1, IKZF2, TFAP2C and TP53. Many studies have provided evidence that these TFs are colsely coupled with TUBB4A in several carcinoma. For example, HOXA1 drives melanoma tumor growth and metastasis involving in diverse cytokine pathways that include the TGF $\beta$ signaling axis. It also down-regulates the expression of microphthalmia-associated transcription factor and other genes required for melanocyte differentiation [37]. Maeda et al. also reported that the expression level of HOXA1 in melanoma with distant metastasis was higher than those in melanoma without it [38]. It is reported that microRNA-214 contributes to melanoma tumor progression through suppression of TFAP2C [39]. Differences in methylation between the primary melanoma lesions and multiple metastases were evident for TFAP2C gene [40]. Tubulin acetylation favors the molecular chaperone Hsp90 recruitment to microtubules, and stimulates the binding and signaling function of the kinase Akt/PKB and transcription factor P53 [41]. Furthermore, Arai et al. reported that the tubulin inhibitor vinca alkaloid enhanced expression of class II beta-tubulin isotype (mTUBB2) in mouse B16F10 melanoma cells via alteration of the tumor suppressor P53 protein [42]. Other studies found that P53 transcriptionally downregulated microtubule-associated protein 4 [43], and that human breast carcinoma cells with a mutated P53 gene displayed an increased level of class IV $\beta$-tubulin $[44,45]$.

We also investigated the downstream factors of TUBB4. Proximity ligation assay (PLA) and immunoprecipitation studies performed on human glioblastoma patient specimen confirm GLUT1 
interaction with TUBB4. Treatment of glioblastoma cells with TUBB4 inhibitor, $C R-42-24$, reduces the expression of GLUT1 however, TUBB4 expression is unaltered upon GLUT1 inhibitor fasentin treatment [14]. Here indicated that GLUT1 may be a downstream target gene of TUBB4A. Also, one of the isoform of Intersectin1 ITSN1-L was able to strengthen cell-cell adhesion by upregulating $\mathrm{N}$-cadherin expression and its re-localization to membrane by ANXA2 and TUBB3/TUBB4 [46].

A limitation of our study mainly lies in that, although enrichment analysis has suggested several related pathways, and extensive literature was conducted to illustrate the mechanism of TUBB4A, there is still a lack of wet-lab experiments to reveal the related celluar mechanism. Despite this limitation, our findings demonstrated the biological function of TUBB4A in SKCM for the first time, suggesting it as a promising prognostic marker and therapeutic target.

\section{Conclusion}

Overall, the findings indicate that TUBB4A functions as a driver factor in increasing melanoma cell proliferation and motility, and the targeted drugs of TUBB4A significantly induced melanoma cell apoptosis and G2/M cell cycle arrest. We would draw the conclusion that TUBB4A may be a potential prognostic marker and therapeutic target of melanoma.

\section{Declarations}

\section{Acknowledgments}

We would like to acknowledge the reviewers for their helpful comments on this paper.

\section{Author contributions}

$\mathrm{JZ}$ did the biological experiments and drafted the manuscript. ZL collected the data and performed the statistical analysis. HL helped to improve the idea and the manuscript. WQ and ZS conceived the main idea and the framework of the manuscript. YC and WG reviewed drafts of the paper. JL and XL supervised the study and provided funding. All authors read and commented on the manuscript.

\section{Funding}

This work was supported by National Natural Science Foundation of China $(82073339 ; 62072058)$, Natural Science Foundation of Jiangsu Province (BK20191157) and Funding of CSCO-Linghang (Y2019AZMS-0002).

\section{Availability of data and material}

The datasets during and/or analyzed during the current study available from the corresponding author on reasonable request. 
Ethics approval and consent to participate

Not applicable.

Consent for publication

All authors agree to publish.

Declaration of interests

The authors declare no competing interests.

\section{References}

1. Schadendorf D, van Akkooi ACJ, Berking C, Griewank KG, Gutzmer R, Hauschild A, Stang A, Roesch A, Ugurel S: Melanoma. The Lancet 2018, 392(10151):971-984.

2. Domingues B, Lopes JM, Soares P, Populo H: Melanoma treatment in review. Immunotargets Ther 2018, 7:35-49.

3. Hessler M, Jalilian E, Xu Q, Reddy S, Horton L, Elkin K, Manwar R, Tsoukas M, Mehregan D, Avanaki K: Melanoma Biomarkers and Their Potential Application for In Vivo Diagnostic Imaging Modalities. Int $J$ Mol Sci 2020, 21(24).

4. Abbas $\mathrm{O}$, Miller DD, Bhawan J: Cutaneous malignant melanoma: update on diagnostic and prognostic biomarkers. Am J Dermatopathol 2014, 36(5):363-379.

5. Piris A, Mihm MC, Jr.: Progress in melanoma histopathology and diagnosis. Hematol Oncol Clin North Am 2009, 23(3):467-480, viii.

6. Eisenstein A, Gonzalez EC, Raghunathan R, Xu X, Wu M, McLean EO, McGee J, Ryu B, Alani RM: Emerging Biomarkers in Cutaneous Melanoma. Mol Diagn Ther 2018, 22(2):203-218.

7. Luke JJ, Flaherty KT, Ribas A, Long GV: Targeted agents and immunotherapies: optimizing outcomes in melanoma. Nat Rev Clin Oncol 2017, 14(8):463-482.

8. Bastian BC: The molecular pathology of melanoma: an integrated taxonomy of melanocytic neoplasia. Annu Rev Pathol 2014, 9:239-271.

9. Aseervatham J: Cytoskeletal Remodeling in Cancer. Biology (Basel) 2020, 9(11).

10. Kavallaris M: Microtubules and resistance to tubulin-binding agents. Nat Rev Cancer 2010, 10(3):194-204.

11. Lee KM, Cao D, Itami A, Pour PM, Hruban RH, Maitra A, Ouellette MM: Class III beta-tubulin, a marker of resistance to paclitaxel, is overexpressed in pancreatic ductal adenocarcinoma and intraepithelial neoplasia. Histopathology 2007, 51(4):539-546.

12. Haider $K$, Rahaman $S$, Yar MS, Kamal A: Tubulin inhibitors as novel anticancer agents: an overview on patents (2013-2018). Expert Opin Ther Pat 2019, 29(8):623-641. 
13. Mandelkow E, Mandelkow EM: Microtubules and microtubule-associated proteins. Curr Opin Cell Biol 1995, 7(1):72-81.

14. Guda MR, Labak CM, Omar SI, Asuthkar S, Airala S, Tuszynski J, Tsung AJ, Velpula KK: GLUT1 and TUBB4 in Glioblastoma Could be Efficacious Targets. Cancers (Basel) 2019, 11(9).

15. Tamura D, Arao T, Nagai T, Kaneda H, Aomatsu K, Fujita Y, Matsumoto K, De Velasco MA, Kato H, Hayashi $\mathrm{H}$ et al: Slug increases sensitivity to tubulin-binding agents via the downregulation of betall and betalVa-tubulin in lung cancer cells. Cancer Med 2013, 2(2):144-154.

16. Ross JA, Vissers JPC, Nanda J, Stewart GD, Husi H, Habib FK, Hammond DE, Gethings LA: The influence of hypoxia on the prostate cancer proteome. Clin Chem Lab Med 2020, 58(6):980-993.

17. Atjanasuppat K, Lirdprapamongkol K, Jantaree P, Svasti J: Non-adherent culture induces paclitaxel resistance in $\mathrm{H} 460$ lung cancer cells via ERK-mediated up-regulation of betalVa-tubulin. Biochem Biophys Res Commun 2015, 466(3):493-498.

18. Tomczak K, Czerwinska P, Wiznerowicz M: The Cancer Genome Atlas (TCGA): an immeasurable source of knowledge. Contemp Oncol (Pozn) 2015, 19(1A):A68-77.

19. Zhang J, Liu H, Zhang W, Li Y, Fan Z, Jiang H, Luo J: Identification of IncRNA-mRNA Regulatory Module to Explore the Pathogenesis and Prognosis of Melanoma. Front Cell Dev Biol 2020, 8:615671.

20. Ritchie ME, Phipson B, Wu D, Hu Y, Law CW, Shi W, Smyth GK: limma powers differential expression analyses for RNA-sequencing and microarray studies. Nucleic Acids Res 2015, 43(7):e47.

21. Yu G, Wang LG, Han Y, He QY: clusterProfiler: an R package for comparing biological themes among gene clusters. OMICS 2012, 16(5):284-287.

22. Vasaikar SV, Straub P, Wang J, Zhang B: LinkedOmics: analyzing multi-omics data within and across 32 cancer types. Nucleic Acids Res 2018, 46(D1):D956-D963.

23. Wishart DS, Feunang YD, Guo AC, Lo EJ, Marcu A, Grant JR, Sajed T, Johnson D, Li C, Sayeeda Z et al: DrugBank 5.0: a major update to the DrugBank database for 2018. Nucleic Acids Res 2018, 46(D1):D1074-D1082.

24. Cabello CM, Lamore SD, Bair WB, 3rd, Qiao S, Azimian S, Lesson JL, Wondrak GT: The redox antimalarial dihydroartemisinin targets human metastatic melanoma cells but not primary melanocytes with induction of NOXA-dependent apoptosis. Invest New Drugs 2012, 30(4):1289-1301.

25. McNeely SC, Taylor BF, States JC: Mitotic arrest-associated apoptosis induced by sodium arsenite in A375 melanoma cells is BUBR1-dependent. Toxicol Appl Pharmacol 2008, 231(1):61-67.

26. Cui R, Widlund HR, Feige E, Lin JY, Wilensky DL, Igras VE, D'Orazio J, Fung CY, Schanbacher CF, Granter SR et al: Central role of p53 in the suntan response and pathologic hyperpigmentation. Cell 2007, 128(5):853-864.

27. Zerfaoui M, Dokunmu TM, Toraih EA, Rezk BM, Abd Elmageed ZY, Kandil E: New Insights into the Link between Melanoma and Thyroid Cancer: Role of Nucleocytoplasmic Trafficking. Cells 2021, 10(2). 
28. Duffy DL, Zhao ZZ, Sturm RA, Hayward NK, Martin NG, Montgomery GW: Multiple pigmentation gene polymorphisms account for a substantial proportion of risk of cutaneous malignant melanoma. $J$ Invest Dermatol 2010, 130(2):520-528.

29. Barrett JH, lles MM, Harland M, Taylor JC, Aitken JF, Andresen PA, Akslen LA, Armstrong BK, Avril MF, Azizi $E$ et al: Genome-wide association study identifies three new melanoma susceptibility loci. Nat Genet 2011, 43(11):1108-1113.

30. Meliso FM, Micali D, Silva CT, Sabedot TS, Coetzee SG, Koch A, Fahlbusch FB, Noushmehr H, Schneider-Stock R, Jasiulionis MG: SIRT1 regulates Mxd1 during malignant melanoma progression. Oncotarget 2017, 8(70):114540-114553.

31. Zheng J, Liu F, Du S, Li M, Wu T, Tan X, Cheng W: Mechanism for Regulation of Melanoma Cell Death via Activation of Thermo-TRPV4 and TRPV2. J Oncol 2019, 2019:7362875.

32. Ravindra KC, Ho WE, Cheng C, Godoy LC, Wishnok JS, Ong CN, Wong WS, Wogan GN, Tannenbaum SR: Untargeted Proteomics and Systems-Based Mechanistic Investigation of Artesunate in Human Bronchial Epithelial Cells. Chem Res Toxicol 2015, 28(10):1903-1913.

33. Imming P, Sinning C, Meyer A: Drugs, their targets and the nature and number of drug targets. Nat Rev Drug Discov 2006, 5(10):821-834.

34. Altonsy MO, Ganguly A, Amrein M, Surmanowicz P, Li SS, Lauzon GJ, Mydlarski PR: Beta3-Tubulin is Critical for Microtubule Dynamics, Cell Cycle Regulation, and Spontaneous Release of Microvesicles in Human Malignant Melanoma Cells (A375). Int J Mol Sci 2020, 21(5).

35. Wawro ME, Sobierajska K, Ciszewski WM, Wagner W, Frontczak M, Wieczorek K, Niewiarowska J: Tubulin beta 3 and 4 are involved in the generation of early fibrotic stages. Cell Signal 2017, 38:2638.

36. Feng C, Song C, Liu Y, Qian F, Gao Y, Ning Z, Wang Q, Jiang Y, Li Y, Li M et al: KnockTF: a comprehensive human gene expression profile database with knockdown/knockout of transcription factors. Nucleic Acids Res 2020, 48(D1):D93-D100.

37. Wardwell-Ozgo J, Dogruluk T, Gifford A, Zhang Y, Heffernan TP, van Doorn R, Creighton CJ, Chin L, Scott KL: HOXA1 drives melanoma tumor growth and metastasis and elicits an invasion gene expression signature that prognosticates clinical outcome. Oncogene 2013, 33(8):1017-1026.

38. Maeda K, Hamada J, Takahashi Y, Tada M, Yamamoto Y, Sugihara T, Moriuchi T: Altered expressions of HOX genes in human cutaneous malignant melanoma. Int J Cancer 2005, 114(3):436-441.

39. Penna E, Orso F, Cimino D, Tenaglia E, Lembo A, Quaglino E, Poliseno L, Haimovic A, Osella-Abate S, De Pittà $C$ et al: microRNA-214 contributes to melanoma tumour progression through suppression of TFAP2C. The EMBO Journal 2011, 30(10):1990-2007.

40. Harbst K, Staaf J, Masback A, Olsson H, Ingvar C, Vallon-Christersson J, Ringner M, Borg A, Jonsson G: Multiple metastases from cutaneous malignant melanoma patients may display heterogeneous genomic and epigenomic patterns. Melanoma Res 2010, 20(5):381-391.

41. Giustiniani J, Daire V, Cantaloube I, Durand G, Pous C, Perdiz D, Baillet A: Tubulin acetylation favors Hsp90 recruitment to microtubules and stimulates the signaling function of the Hsp90 clients 
Akt/PKB and p53. Cell Signal 2009, 21(4):529-539.

42. Arai K, Matsumoto $Y$, Nagashima $Y$, Yagasaki K: Regulation of class II beta-tubulin expression by tumor suppressor p53 protein in mouse melanoma cells in response to Vinca alkaloid. Mol Cancer Res 2006, 4(4):247-255.

43. Zhang CC, Yang JM, Bash-Babula J, White E, Murphy M, Levine AJ, Hait WN: DNA damage increases sensitivity to vinca alkaloids and decreases sensitivity to taxanes through p53-dependent repression of microtubule-associated protein 4. Cancer Res 1999, 59(15):3663-3670.

44. Galmarini CM, Kamath K, Vanier-Viornery A, Hervieu V, Peiller E, Falette N, Puisieux A, Ann Jordan M, Dumontet $C$ : Drug resistance associated with loss of $p 53$ involves extensive alterations in microtubule composition and dynamics. Br J Cancer 2003, 88(11):1793-1799.

45. Galmarini CM, Falette N, Tabone E, Levrat C, Britten R, Voorzanger-Rousselot N, Roesch-Gateau O, Vanier-Viornery A, Puisieux A, Dumontet C: Inactivation of wild-type p53 by a dominant negative mutant renders MCF-7 cells resistant to tubulin-binding agent cytotoxicity. Br J Cancer 2001, 85(6):902-908.

46. Shao Y, Chong W, Liu X, Xu Y, Zhang H, Xu Q, Guo Z, Zhao Y, Zhang M, Ma Y et al: Alternative splicingderived intersectin1-L and intersectin1-S exert opposite function in glioma progression. Cell Death Dis 2019, 10(6):431.

\section{Tables}

Table 1. GEO datasets enrolled in the study

\begin{tabular}{llll} 
Database & \multicolumn{2}{c}{ Sample } & Platform \\
\cline { 2 - 3 } & Normal & Melanoma & \\
\hline GSE46517 & 8 & 104 & Affymetrix U133A \\
\hline GSE15605 & 16 & 58 & Affymetrix U133 Plus 2.0 \\
\hline GSE3189 & 7 & 45 & Affymetrix U133A
\end{tabular}

Table 2. Genes significantly related to the prognosis of patients with melanoma 


\begin{tabular}{|lll|}
\hline Gene Symbol & \multicolumn{2}{l|}{$P$ value } \\
\cline { 2 - 3 } & OS & RFS \\
\hline TUBB4A & 0.000039 & 0.013 \\
\hline PSEN2 & 0.0017 & 0.028 \\
\hline SLC45A2 & 0.002 & 0.048 \\
\hline QPRT & 0.03 & 0.016 \\
\hline TRPV2 & 0.039 & 0.016 \\
\hline
\end{tabular}

Figures 
A

B

GSE46517

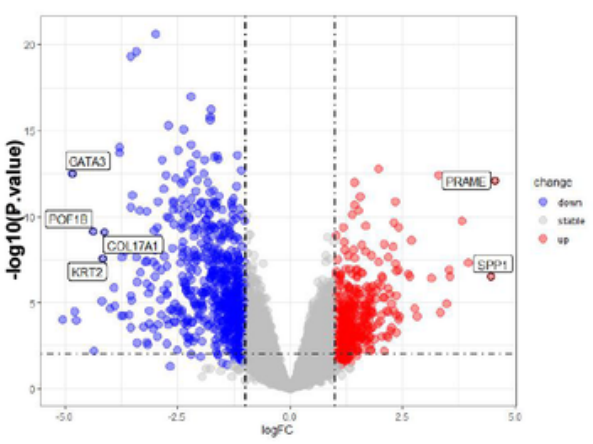

D
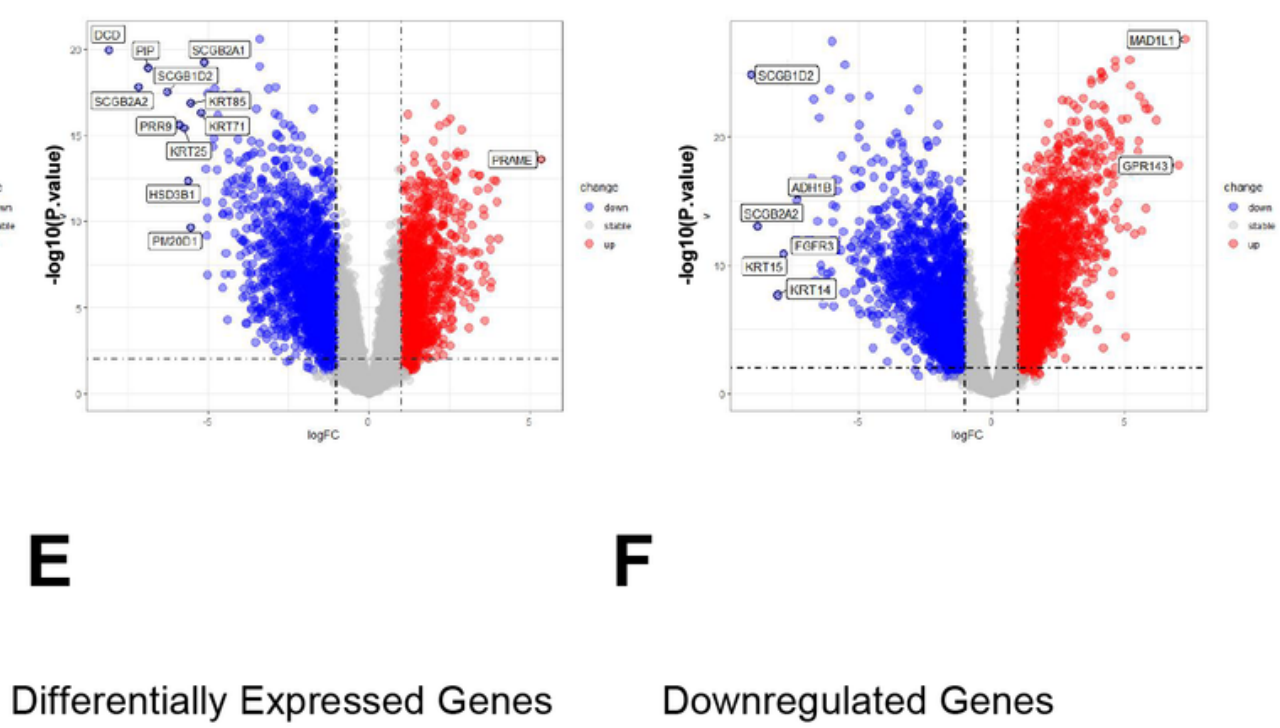

E

F

Downregulated Genes

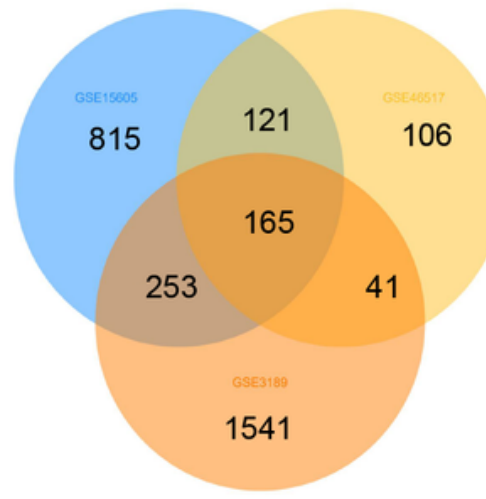

G

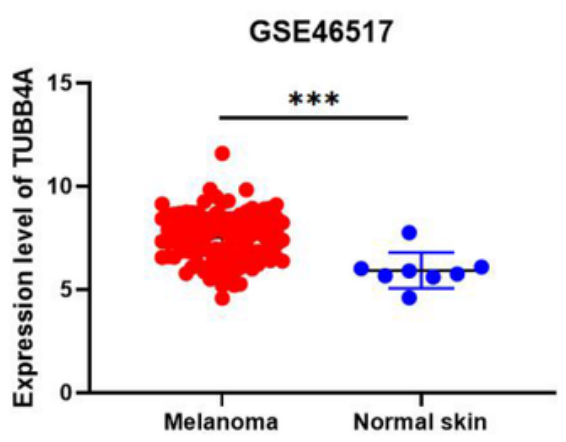

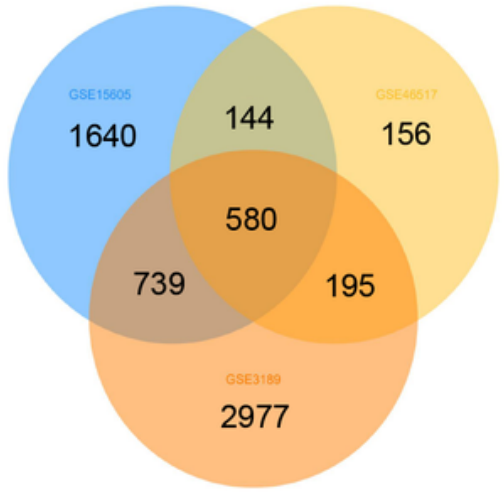

H

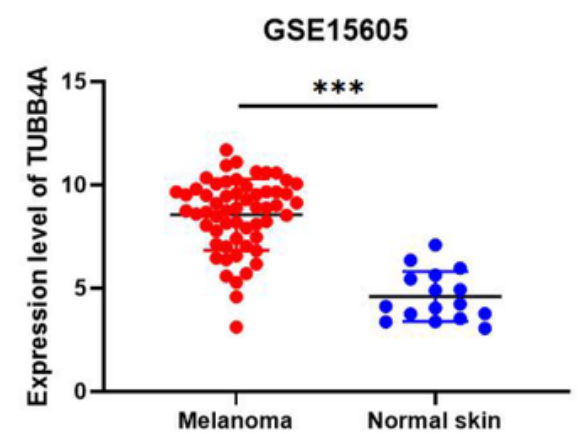

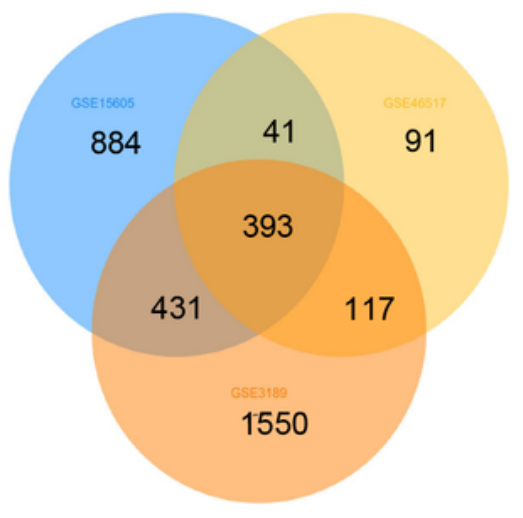

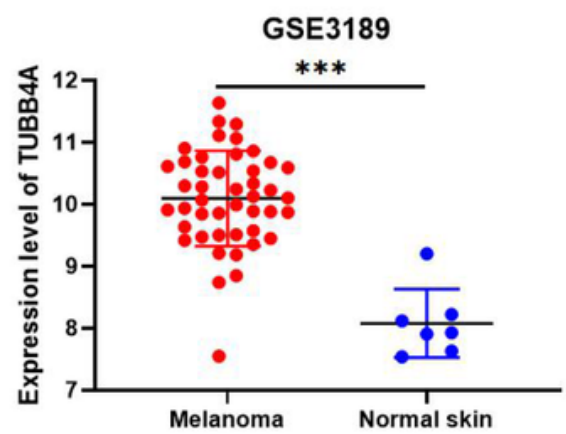

\section{Figure 1}

Differentially expressed genes between normal samples and melanoma patients (fold change $>2$, P-value $<0.05$ ). A-C Volcano plots of the P-value as a function of weighted fold change for DEGs, red dots represent significantly upregulated expressed genes and blue dots represent significantly downregulated expressed genes. D-F Overlapping analyses of DEGs in each dataset. Every circle corresponded to a dataset. The numbers of DEGs in each overlapped area were marked in relevant position. DEGs within 
three data sets were regarded as credible in each Venn diagram. G-I mRNA expression of TUBB4A in melanoma and normal tissues in GEO dataset.

A

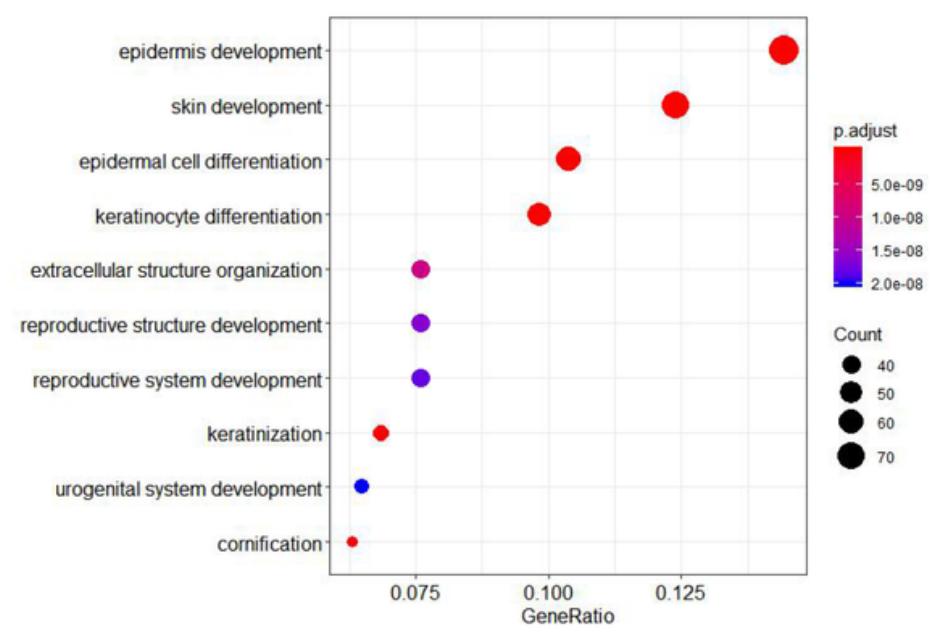

C

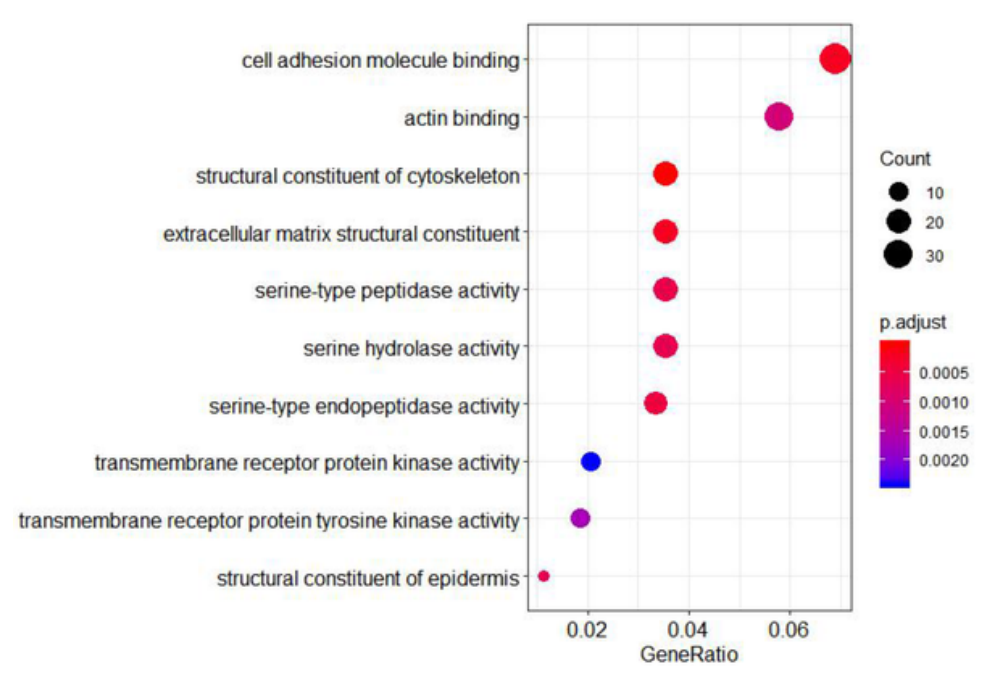

B

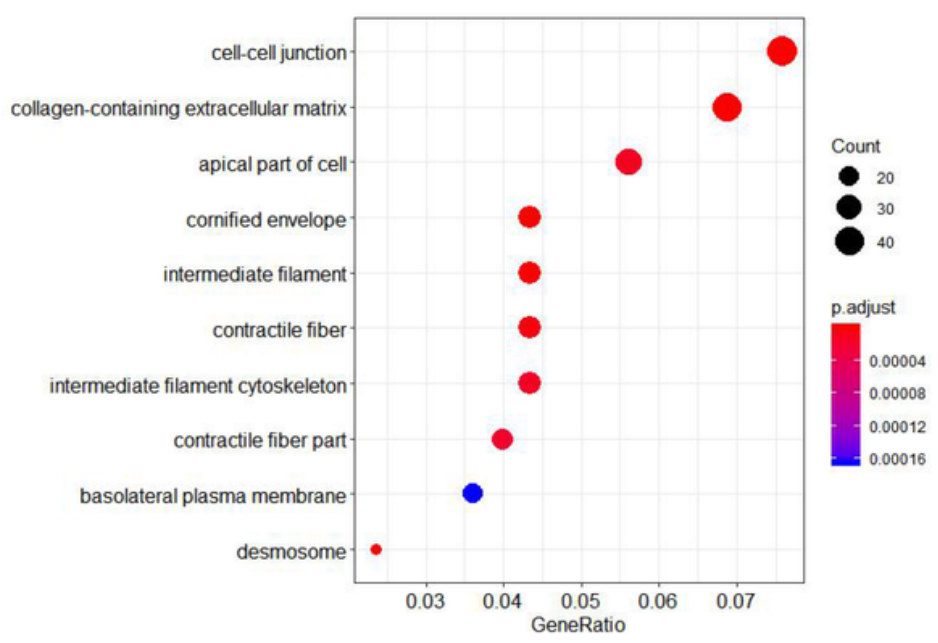

D

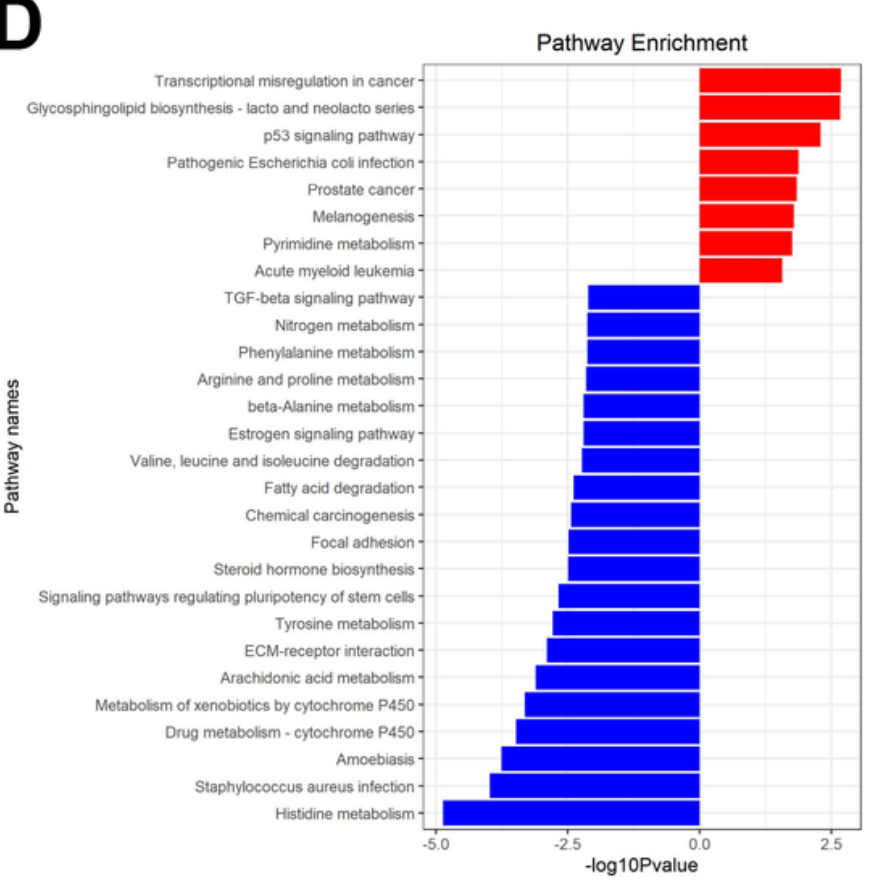

Figure 2

GO and KEGG pathway enrichment analysis. A-C GO biological processes (BP), cellular composition (CC), and molecular function (MF)enrichment analysis of DEGs. D KEGG pathway analysis of DEGs. 
A
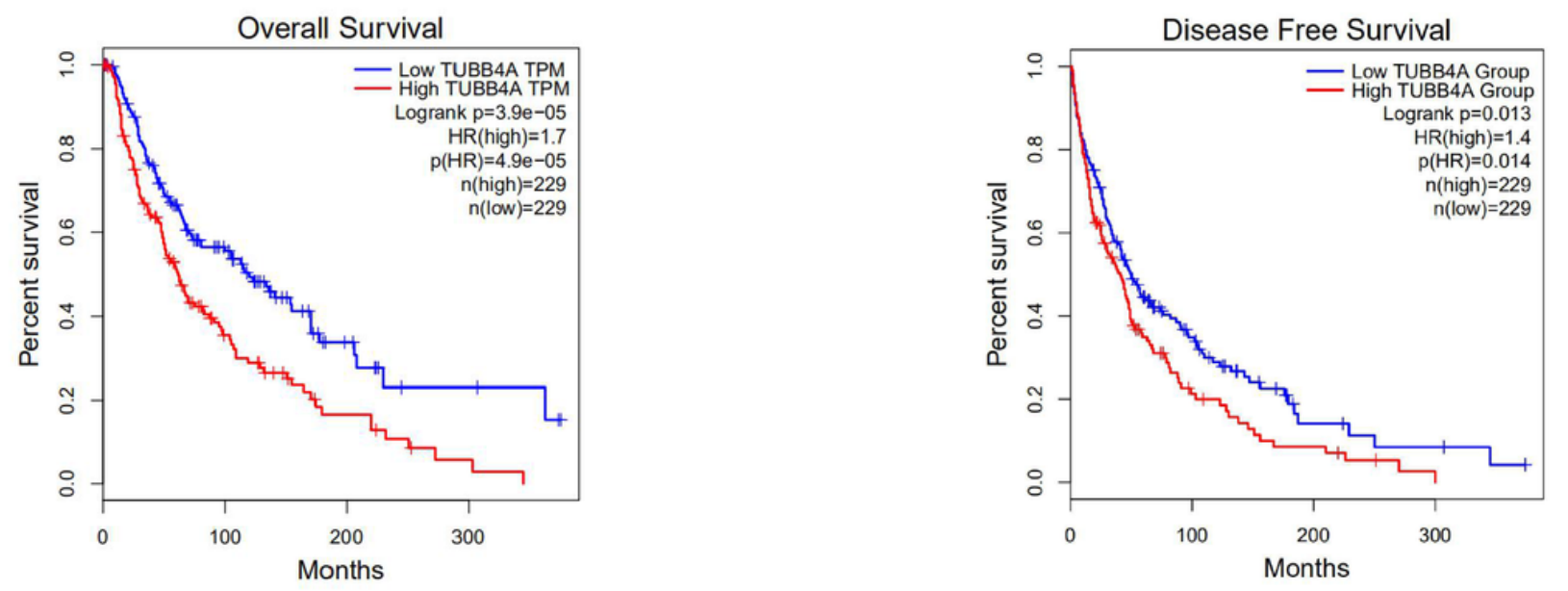

C

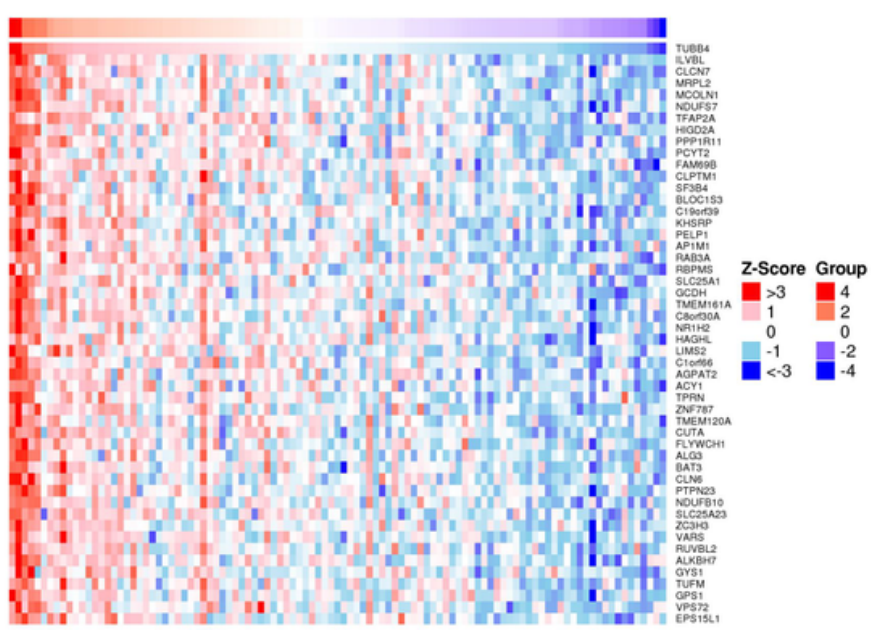

D

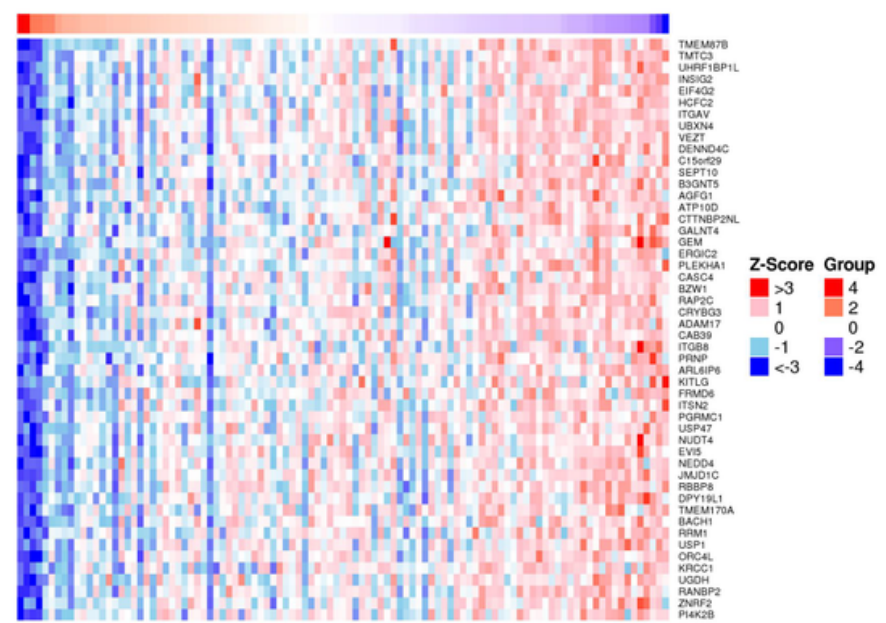

\section{Figure 3}

Survival and co-expression analysis. A, B OS and DFS survival curve of TUBB4A with the significant logrank P-value. C, D Heat maps show the top 50 significant genes positively and negatively correlated with TUBB4A in SKCM. Red indicates positively correlated genes, and blue indicates negatively correlated genes. 

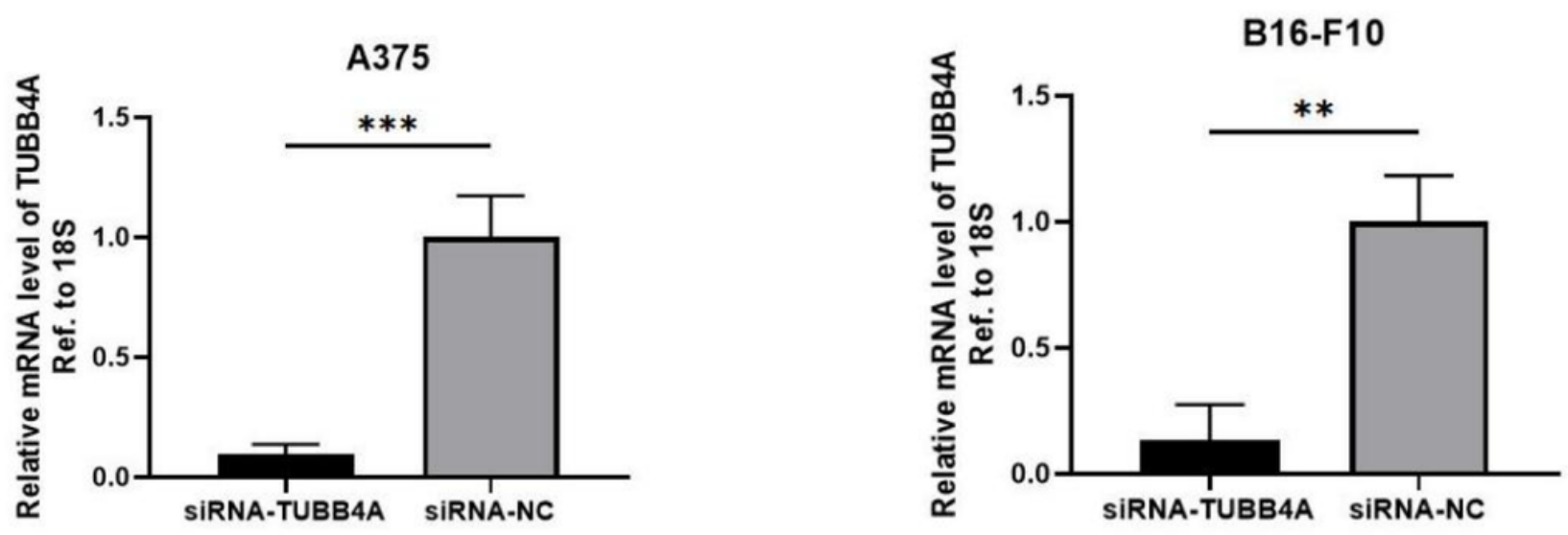

C

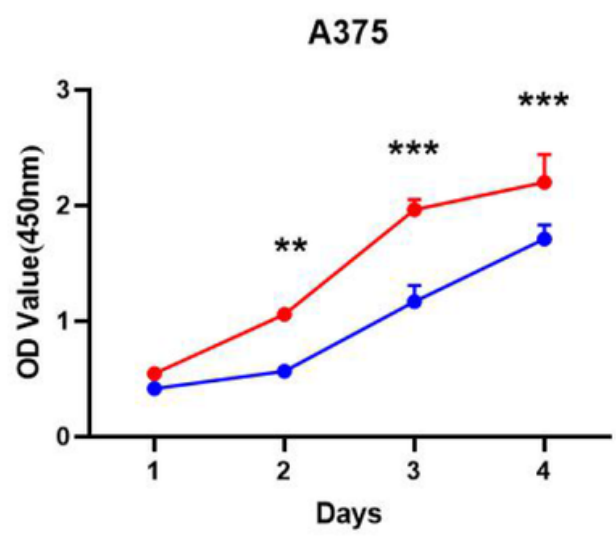

D

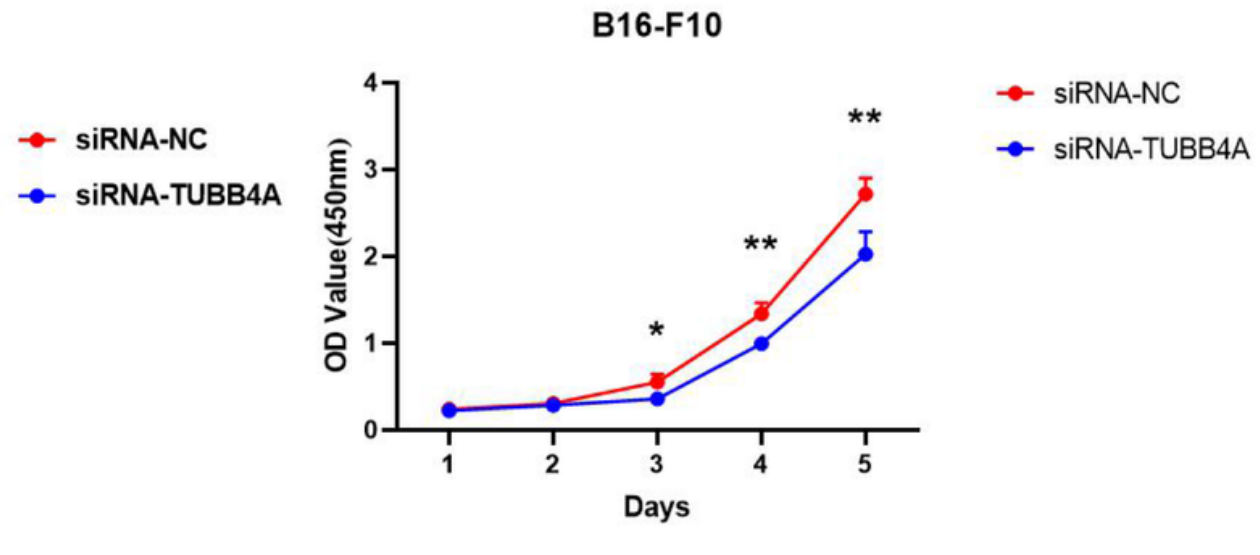

Figure 4

TUBB4A knockdown suppressed proliferation of SKCM cells. A, B Knockdown of TUBB4A was confirmed by qRT-PCR analysis in A375 and B16-F10 cells. C, D CCK8 cell proliferation assays in A375 and B16-F10 cells with or without TUBB4A knockdown. ${ }^{\star} P<0.05$, ${ }^{\star *} \mathrm{P}<0.01$, ${ }^{\star \star *} \mathrm{P}<0.001$. 


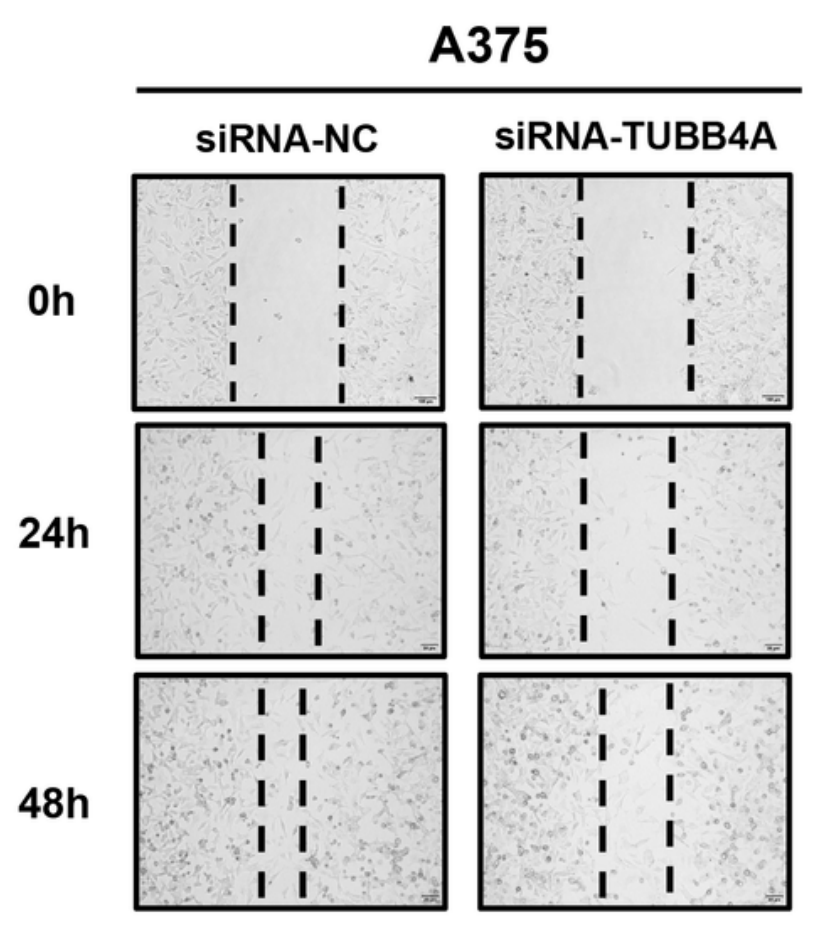

C

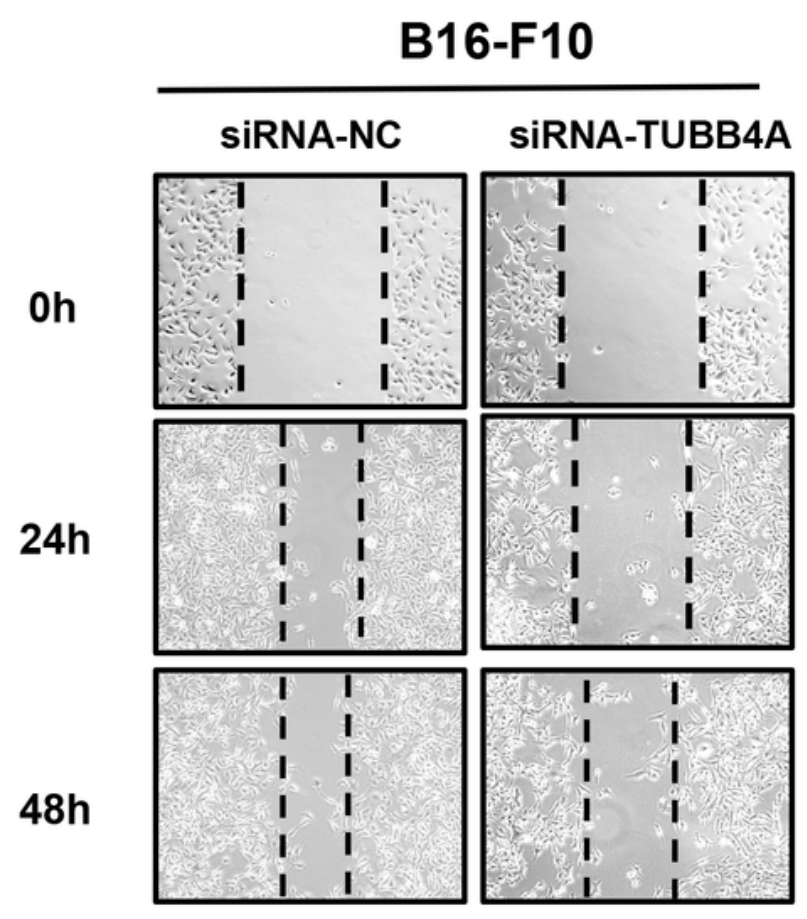

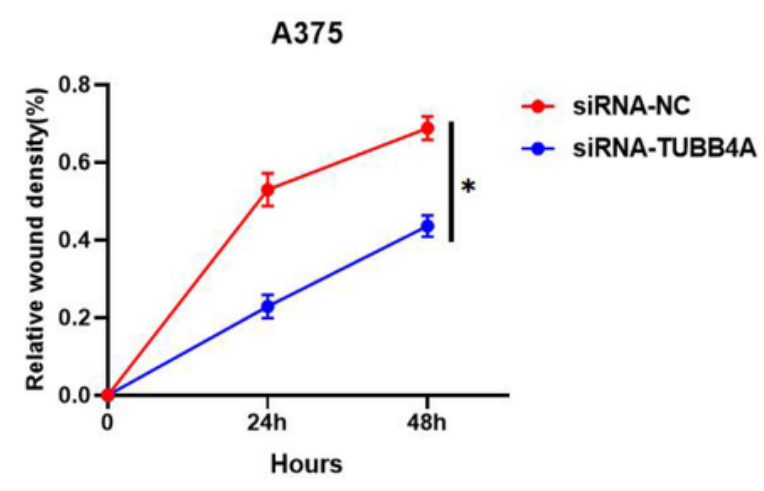

D

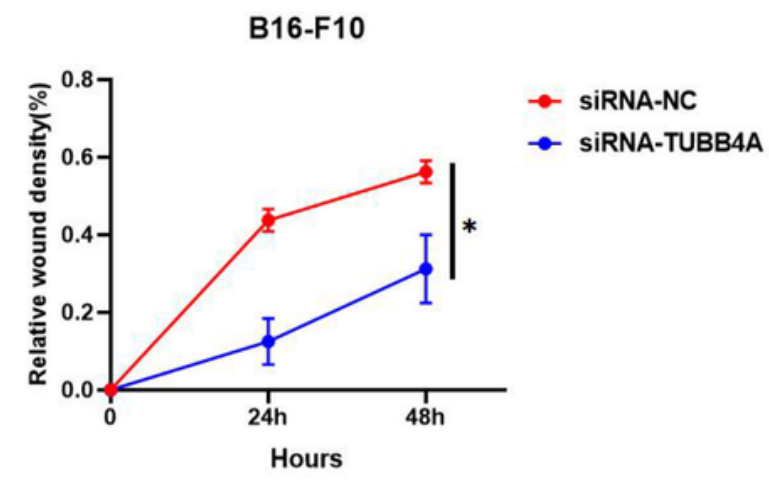

\section{Figure 5}

TUBB4A knockdown suppressed migration of SKCM cells by wound healing assay. Scratch wound healing assay was applied in A375 (A, B) and B16-F10 (C, D) cells with or without TUBB4A knockdown. 


\section{A375}
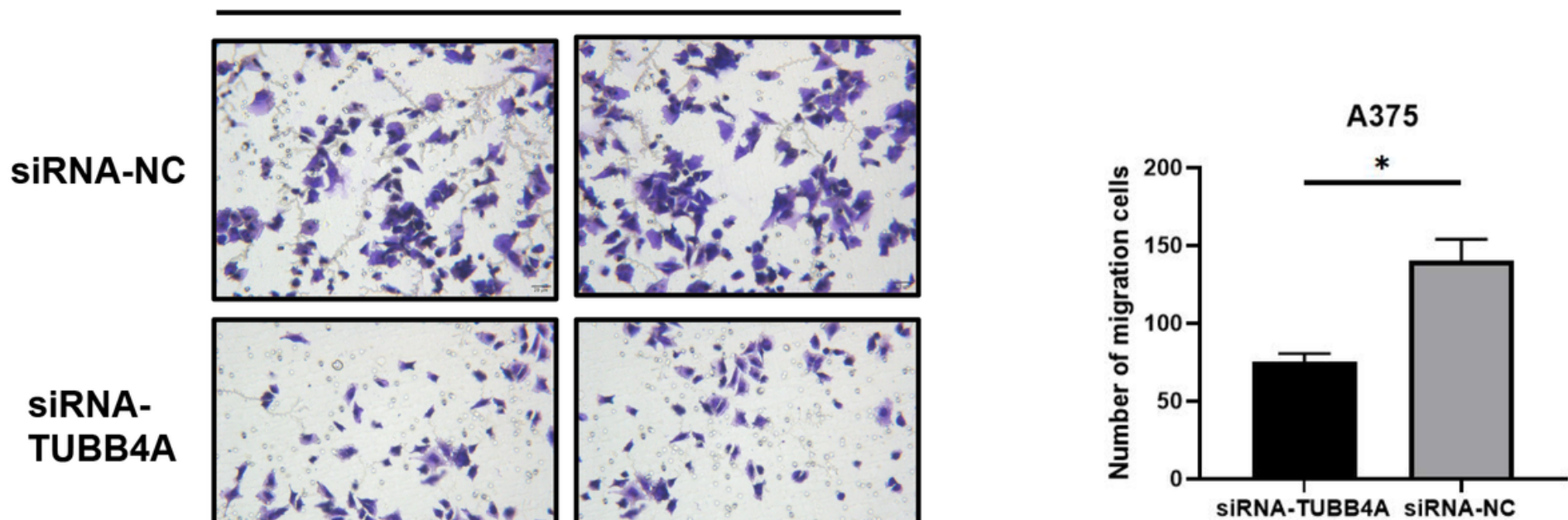

SiRNATUBB4A
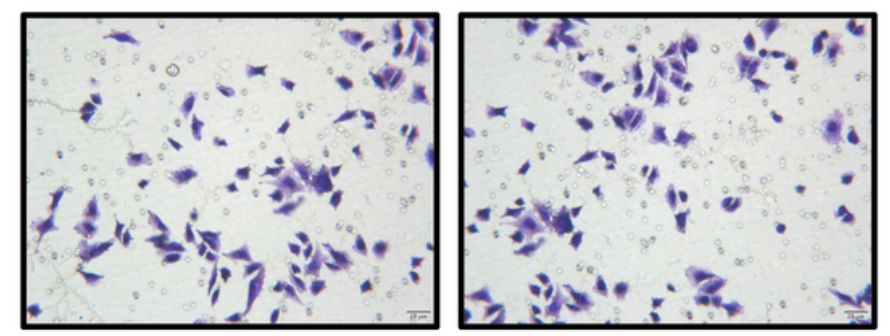

\section{B16-F10}

siRNA-NC
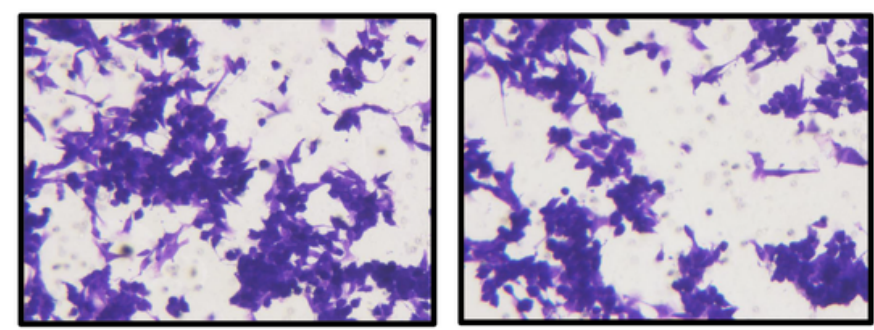

SiRNATUBB4A
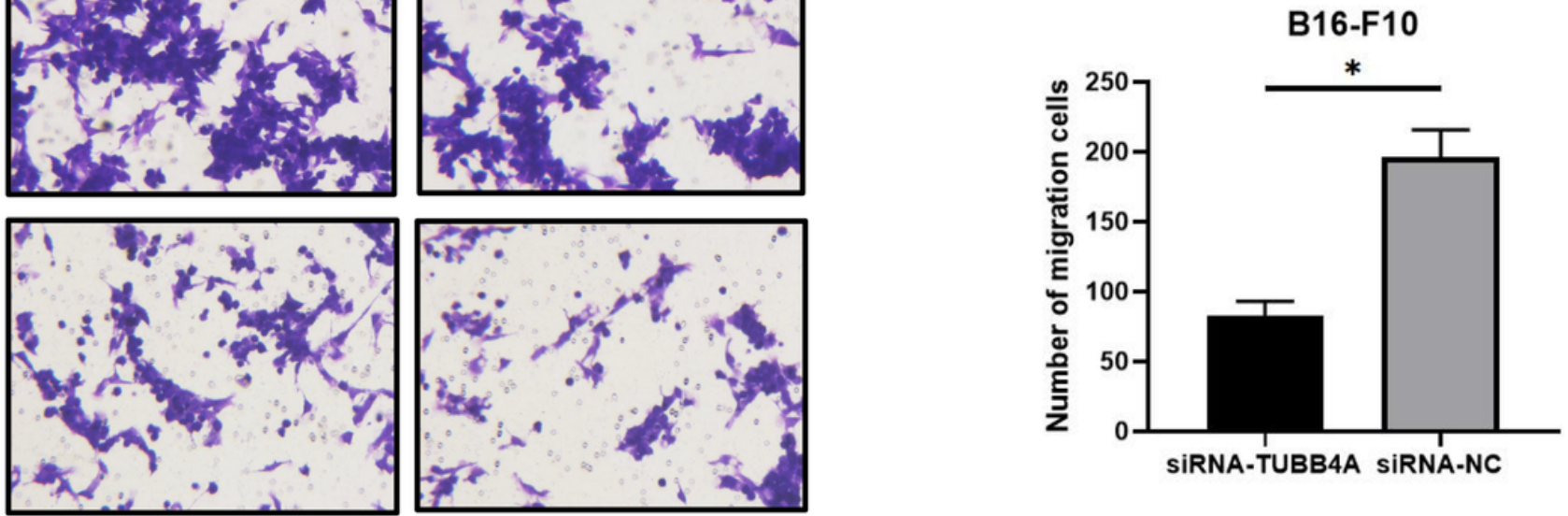

Figure 6

TUBB4A knockdown suppressed migration of SKCM cells by transwell assay. Transwell assay was applied in A375 (A, B) and B16-F10 (c, d) cells with or without TUBB4A knockdown. ${ }^{\star} \mathrm{P}<0.05,{ }^{*} \mathrm{P}<0.01$, $\star \star \star P<0.001$. 
A375
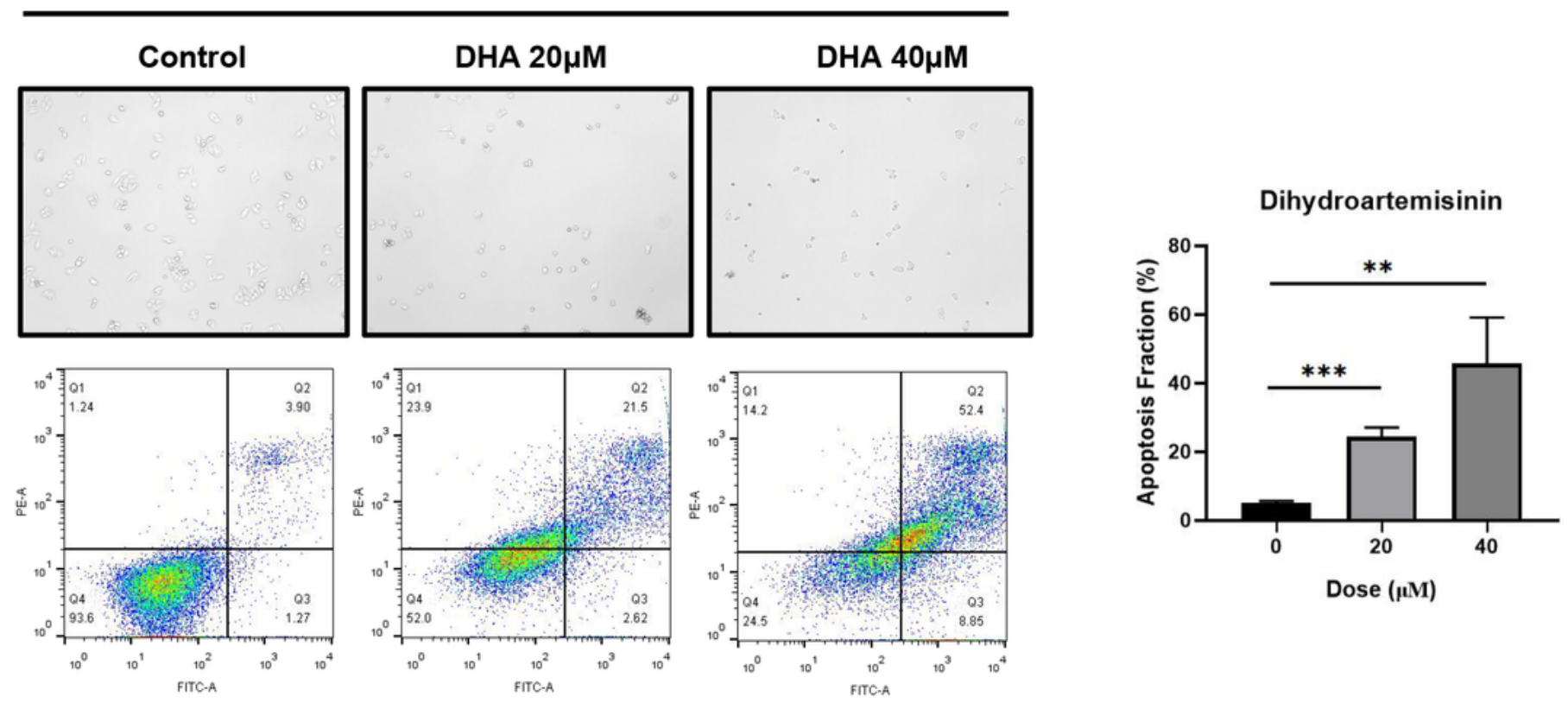

C
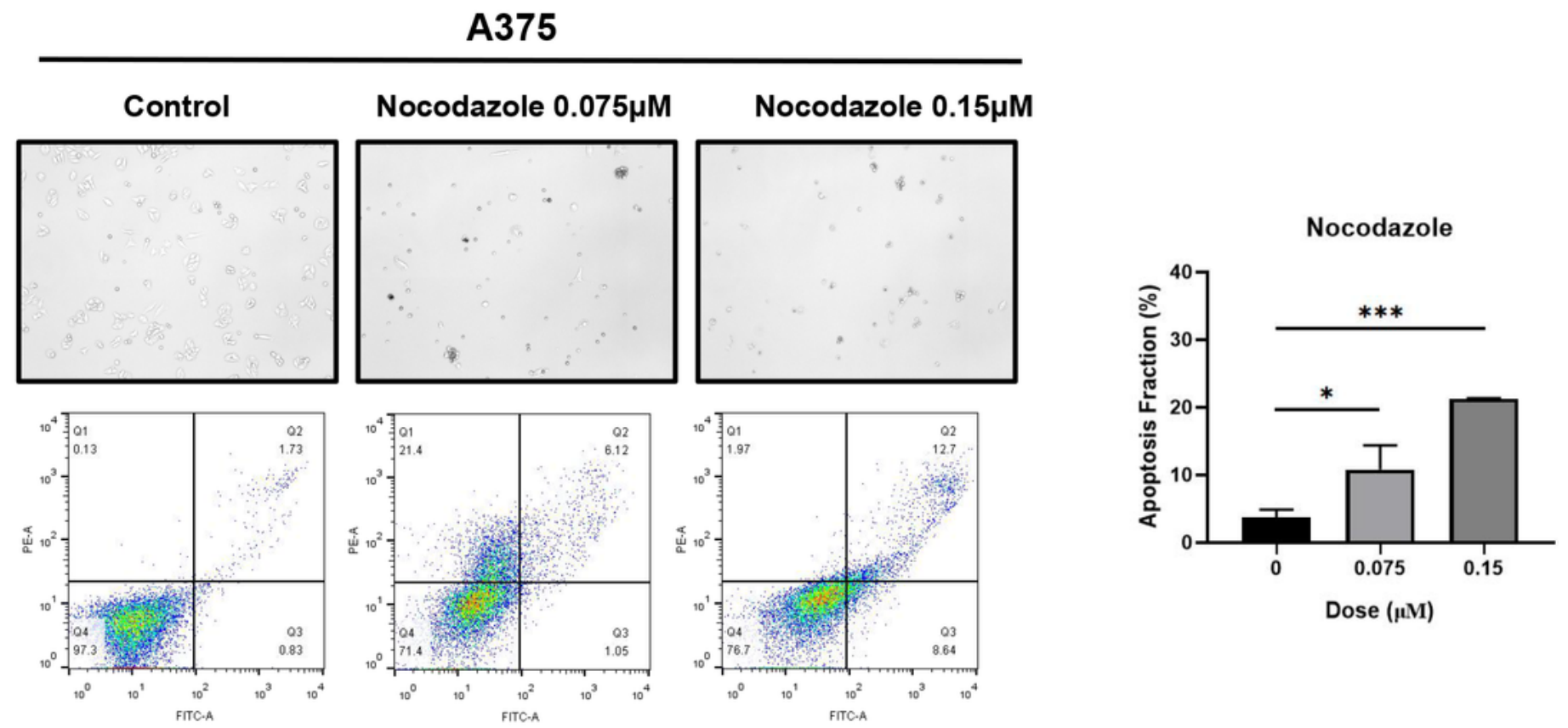

Figure 7

Induction of apoptosis in A375 cells after exposure to Dihydroartemisinin (DHA) and Nocodazole. A, B Representative apoptosis photograph of $A 375$ cells treated with or without DHA (20 or $40 \mu \mathrm{M})$. C, D Representative apoptosis photograph of $A 375$ cells treated with or without Nocodazole ( 0.075 or $0.15 \mu \mathrm{M})$. Flow cytometry analysis of A375 cell apoptosis by double staining with annexin (An) V-FITC 
and PI. Quadrant Q1 represents An-/PI+ necrotic cells, Q2 - An+/ Pl+late apoptotic cells, Q3 - An-/PI viable cells, Q4 - An+/PI early apoptotic cells. ${ }^{*} \mathrm{P}<0.05$, ${ }^{\star *} \mathrm{P}<0.01$, ***P $<0.001$.

A
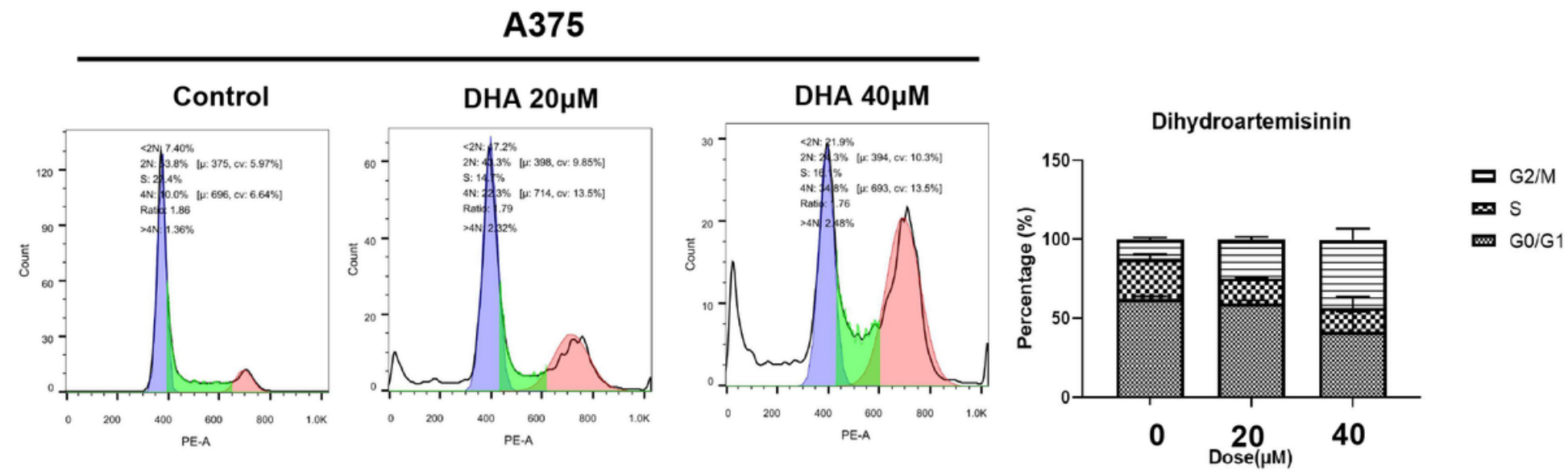

C

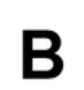

\author{
|
}

